\title{
A numerical investigation of coorbital stability and libration in three dimensions
}

\author{
M. H. M. Morais ${ }^{1}$ (D) F. Namouni ${ }^{2}$
}

Received: 7 November 2015 / Revised: 27 January 2016 / Accepted: 13 February 2016 /

Published online: 9 March 2016

C) Springer Science+Business Media Dordrecht 2016

\begin{abstract}
Motivated by the dynamics of resonance capture, we study numerically the coorbital resonance for inclination $0 \leq I \leq 180^{\circ}$ in the circular restricted three-body problem. We examine the similarities and differences between planar and three dimensional coorbital resonance capture and seek their origin in the stability of coorbital motion at arbitrary inclination. After we present stability maps of the planar prograde and retrograde coorbital resonances, we characterize the new coorbital modes in three dimensions. We see that retrograde mode I (R1) and mode II (R2) persist as we change the relative inclination, while retrograde mode III (R3) seems to exist only in the planar problem. A new coorbital mode (R4) appears in 3D which is a retrograde analogue to an horseshoe-orbit. The Kozai-Lidov resonance is active for retrograde orbits as well as prograde orbits and plays a key role in coorbital resonance capture. Stable coorbital modes exist at all inclinations, including retrograde and polar obits. This result confirms the robustness the coorbital resonance at large inclination and encourages the search for retrograde coorbital companions of the solar system's planets.
\end{abstract}

Keywords Co-orbital resonance $\cdot$ Resonance $\cdot$ Three-body problem $\cdot$ Retrograde resonances $\cdot$ Kozai-Lidov mechanism $\cdot$ Resonance trapping

\section{Introduction}

The recent discovery of the Centaurs and Damocloids 2006 BZ8, 2008 SO218, 2009 QY6 and 1999 LE31 in retrograde resonance with Jupiter and Saturn (Morais and Namouni 2013a) has shown that retrograde resonances although weaker than their prograde counterparts (Morais and Namouni 2013b) (hereafter Paper I) are capable of temporarily trapping minor bodies for a few thousand years at a time. A subsequent statistical study of resonance capture at

\footnotetext{
M. H. M. Morais

helena.morais@rc.unesp.br

1 Instituto de Geociências e Ciências Exatas, Universidade Estadual Paulista, Av. 24-A, 1515, Rio Claro, SP 13506-900, Brazil

2 Université Côte d'Azur, CNRS, Observatoire, de la Côte d'Azur, CS 34229, 06304 Nice, France
} 
arbitrary inclination (Namouni and Morais 2015) (hereafter Paper II) reported the unexpected result that although weaker than prograde resonances, retrograde resonances have a higher capture likelihood. Since resonance provides a protection mechanism against disruptive close encounters with the planets, the current Centaurs and Damocloids in retrograde resonance could be among the dynamically oldest minor bodies in the outer solar system. It is therefore of interest to study closely the dynamics of such resonances in terms of both capture and stability. In this regard, the strongest resonance for retrograde motion is the coorbital (1:1) resonance. Moreover, it was shown in Paper II how inwardly drifting outer particles that encounter Jupiter's web of mean motion resonances with a small relative inclination are captured with great likelihood in the coorbital resonance if their orbits are retrograde, and in the 1:2 resonance if their orbits are prograde. For this reason, in this paper we investigate further the workings of capture and stability in the retrograde coorbital resonance.

In Sect. 2 we start by reviewing our work presented in Paper I on the coorbital resonant angles at arbitrary inclination and the possible coorbital modes in 2D. We progress by examining the similarities and differences between capture in the planar and three dimensional configurations (Sect. 3) as dynamical models of coorbital motion often rely on the planar problem to access the basics of resonance capture. We show that the two configurations are fundamentally different owing to the presence of the Kozai resonance in $3 \mathrm{D}$ that conditions the three-stage capture process reported in Paper II. To further understand how specific librations are possible in the planar and three-dimensional configurations, we produce $(2 \mathrm{D}$ and 3D) stability maps (in Sects. 4, 5) for a wide range of eccentricities $[0,1]$ as well as a wide range of inclinations $\left[0,180^{\circ}\right]$. These maps enable us to access the similarities and differences between prograde and retrograde dynamical stability. Our conclusions about the dynamics of capture and stability in the coorbital resonance are given in Sect. 6.

\section{Critical angles of the coorbital resonance at arbitrary inclination}

Classical expansions of the disturbing function of the circular restricted 3-body problem $(\mathrm{CR} 3 \mathrm{BP})^{1}$ have been developed to model prograde nearly coplanar motion (relative inclination $I \approx 0$ ). For instance, Laplace's literal expansion of the prograde disturbing function (Murray and Dermott 1999) involves powers of the small parameter $\sin ^{2}(I / 2)$ hence it is invalid for retrograde nearly coplanar motion $\left(I \approx 180^{\circ}\right)$. As explained in Paper I the expansion of the retrograde disturbing function should be done in powers of the small parameter $\cos ^{2}(I / 2)$, or equivalently, by applying the following canonical transformation to the expansion of the prograde disturbing function: ${ }^{2}$

$$
I^{\star}=180^{\circ}-I \quad \lambda_{\mathrm{J}}^{\star}=-\lambda_{\mathrm{J}} \quad \omega^{\star}=\omega-\pi \quad \Omega^{\star}=-\Omega-\pi .
$$

From Eq. 1 we obtain the transformations between longitudes of pericentre $\varpi^{\star}=\varpi-2 \Omega$ and mean longitudes $\lambda^{\star}=\lambda-2 \Omega$. When $I=180^{\circ}$ (planar retrograde case) the line of nodes is undefined hence $\varpi^{\star}=\varpi$ and $\lambda^{\star}=\lambda$.

As shown in Paper I this method allows to identify the terms associated with a $p: q$ retrograde resonance which appear at order $p+q$ in the small parameters $e$ and $\cos (I / 2)$, in contrast with the terms associated with a $p: q$ prograde resonance that appear at order

\footnotetext{
1 We use standard Keplerian elements for the test particle's orbit: $a$ (semi-major axis), $e$ (eccentricity), $I$ (inclination), $f$ (true anomaly), $\omega$ (argument of pericentre), $\Omega$ (longitude of pericentre), $M$ (mean anomaly), $\lambda$ (mean longitude). The planet's circular orbit has semi-major axis $a_{\mathrm{J}}=1$ and mean longitude $\lambda_{\mathrm{J}}$.

2 This is equivalent to obtaining a retrograde configuration of mutual inclination $I>90^{\circ}$ from a prograde configuration of mutual inclination $I<90^{\circ}$ by inverting the motion of the planet with respect to the star.
} 
$p-q$ in the small parameters $e$ and $\sin (I / 2)$. In particular, when $p=q=1$ the lowest order terms in the retrograde expansion are proportional to $e^{2} \cos \left(\lambda^{\star}+\lambda_{\mathrm{J}}^{\star}-2 \varpi^{\star}\right)$ and $\cos ^{2}(I / 2) \cos \left(\lambda^{\star}+\lambda_{\mathrm{J}}^{\star}-2 \Omega^{\star}\right)$, while the lowest order terms in the prograde expansion are proportional to $\cos \left(\lambda-\lambda_{\mathrm{J}}\right)$ and $e^{2} \sin ^{2}(I / 2) \cos \left(\lambda-\lambda_{\mathrm{J}}-2 \varpi+2 \Omega\right)$. Moreover, written in terms of standard orbital elements, the angles with $p=q=1$ that appear at 2 nd order in the retrograde expansion are $\phi^{\star}=\lambda-\lambda_{\mathrm{J}}-2 \varpi+2 \Omega$ and $\phi=\lambda-\lambda_{\mathrm{J}}$, associated with terms $\mathcal{O}\left(e^{2}\right)$ and $\mathcal{O}\left(\cos ^{2}(I / 2)\right)$, respectively. When $I=180^{\circ}$ there is a single angle $\phi^{\star}$ and when $I=0$ there is a single angle $\phi$.

Expansions of the disturbing function do not converge when the semi-major ratio $\alpha \rightarrow 1$ and consequently are not appropriate to model the coorbital resonance which is best studied semi-analytically (Namouni 1999; Namouni et al. 1999; Nesvorný et al. 2002). The angles $\phi$ and $\phi^{\star}$ appear naturally in the expansion-free expression of the averaged Hamiltonian of the $3 \mathrm{D}$ coorbital problem (Paper I). When $I=0$ or $I=180^{\circ}$ there is a single angle $\left(\phi\right.$ or $\left.\phi^{\star}\right)$, in agreement with the analysis presented above, making the $2 \mathrm{D}$ (retrograde or prograde) coorbital problems integrable. In Paper I we constructed surfaces of section of the planar CR3BP exploring the phase-space in the vicinity of the major retrograde resonances. These showed that in 2D there are three possible retrograde coorbital modes: $\phi^{\star}$ librating around 0 which occurs at a wide range of eccentricities (mode I or R1); $\phi^{\star}$ librating around $180^{\circ}$, at large eccentricity (mode II or R2) or small eccentricity (mode III or R3). These resonant orbits are, as expected, associated with the equilibria of the 2D retrograde coorbital Hamiltonian (Paper I).

Figure 1 shows planar retrograde coorbitals in the vicinity of the possible resonant centers viewed in the synodic frame. These were obtained by numerical integration of the CR3BP's equations of motion with mass ratio $\mu=0.001$ and unit distance between star and planet. Mode $\mathrm{R} 1$ orbits are stable at a wide range of eccentricities, from high $e$ values (Fig. 1: top left) limited by the collision with the star at $e \approx 1$, down to low $e$ values (Fig. 1: top right) limited by the collision separatrix ${ }^{3}$ located at pericentre and apocentre. Mode R 2 orbits are only possible at high eccentricity (Fig. 1: low left) and the lower limit on the eccentricity is set by the collision separatrix located halfway between pericentre and apocentre. Mode R3 orbits only occur at small eccentricity and are stable over long integration times despite having close encounters to the planet halfway between pericentre and apocentre.

There are three possible 2D prograde coorbital modes: $\phi$ librating around 0 (retrogradesatellite orbits: ${ }^{4} \mathrm{RS}$ ), $\pm 60^{\circ}$ (tadpole orbits: T) or $180^{\circ}$ (horseshoe orbits: H).

Coorbital motion in 3D has been studied only in the case of prograde inclination (Namouni 1999; Namouni et al. 1999; Nesvorný et al. 2002). Retrograde planar coorbital modes have been recently discovered (Paper I) hence it is expected that at least some of these also exist when $90^{\circ}<I<180^{\circ}$. The purpose of this article is to study numerically the case of retrograde inclination and to relate the retrograde coorbital modes that appear in $2 \mathrm{D}$ and $3 \mathrm{D}$ with recent results regarding capture of retrograde orbits in the coorbital resonance (Paper II).

\section{Coorbital capture of retrograde orbits: 2D versus 3D}

Capture of a drifting particle in the Jupiter-Sun three-body problem was examined in Paper I in terms of statistics as function of the particle's initial eccentricity, inclination and the planet's

\footnotetext{
3 The chaotic region due to the cumulative effect of disruptive close encounters with the planet.

4 Also known as quasi-satellite orbits (Mikkola et al. 2004). These have clockwise motion around the planet in the synodic frame and are not true satellites since the orbits are bound to the star.
} 
Fig. 1 Orbits in the planar retrograde coorbital resonance at mass ratio $\mu=0.001$ seen in synodic frame. Initial $\phi^{\star}=0$ and $a=1.0, e=0.9$ (top left: mode I or R1); $a=1.0450685, e=0.12$ (top right: mode I or R1). Initial $\phi^{\star}=180^{\circ}$ and $a=1.0, e=0.9$ (low left: mode II or R2), $a=1.0138672, e=0.2$ (low right: mode III or R3). The star (Sun) is located at $(0,0)$ and the planet (Jupiter) at $(1,0)$. The gray circle of unit radius helps locate the inner and outer parts of the resonant obits. The pericenter and apocenter of the orbits are the points with $y=0$ and $\dot{x}=0$. The minimum distance to the planet of mode $\mathrm{R} 3$ orbit (low right panel) is larger than 0.01 and the orbit is stable over long integration times
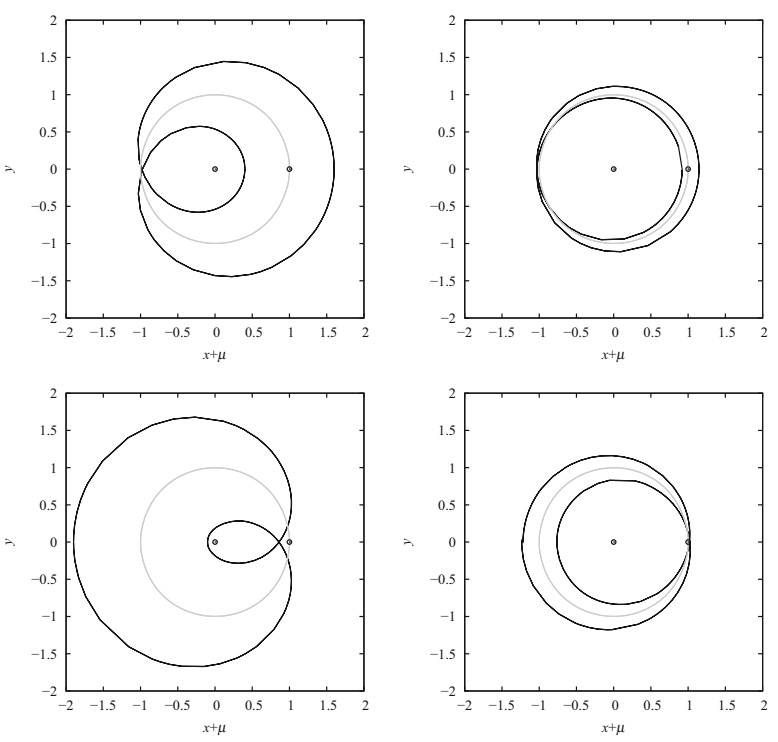

eccentricity. In the following we examine closely capture in the retrograde coorbital resonance and show that its dynamics is richer than previously thought. In particular, extrapolating the workings of the capture process from the planar problem is not adequate to understand the dynamics in three dimensions.

We first start by recalling how capture proceeds in the planar retrograde problem and consider a particle that drifts from a semi-major axis equal to 1.2 units of Jupiter's orbit semi-major axis with both bodies on initially circular orbits. Orbital drift is modeled by a drag force of the type $-k \mathbf{v}$ where $k$ is the friction coefficient and $\mathbf{v}$ the particle's velocity. This implies a semi-major axis function $a(t)=a(0) \exp (-2 k t)$ with a characteristic drift time $(2 k)^{-1}$ that we set to $8 \times 10^{5}$ planetary orbital periods. Resonance crossing is therefore adiabatic and the initial semi-major axis close to the planet is set to maximize the likelihood of capture in the coorbital resonance. For the planar configuration, it is simpler to use the convention adopted by Morais and Giuppone (2012) hence we integrate the 2D equations of motion with negative mean motion of the planet. The planar coorbital retrograde resonance argument is $\phi^{\star}=\lambda+\lambda_{\mathrm{J}}-2 \varpi$ where $\dot{\lambda}_{\mathrm{J}}<0$ due to the planet's negative mean motion, $\lambda$ and $\varpi$ are the standard mean longitude and longitude of perihelion. Figure 2 shows that capture in the coorbital mode with center $\phi^{\star}=180^{\circ}$ (mode R3) occurs at around $1.3 \times 10^{5}$ orbital periods when the particle is inside Jupiter's Hill sphere of radius 0.0693. According to Paper II, 2D initial circular orbits are captured with unit probability in mode R3. Close approaches to the planet's collision separatrix (Namouni 1999) result in large semi-major axis and eccentricity perturbations that ultimately eject the particle from the planet's vicinity.

In order to reveal the different dynamical processes at work with respect to the planar problem we repeat this simulation integrating the $3 \mathrm{D}$ equations of motion where the mean motion of the planet is positive and the the test particle has initial inclination $179.999^{\circ}$. In three dimensions, a retrograde orbit has inclination $180^{\circ}>I>90^{\circ}$ and the convention of Paper I and Sect. 2 for the angles is used. The results are shown in Fig. 3. Unlike the planar configuration, capture occurs outside the Hill sphere (of radius $R_{\mathrm{H}}=0.0693$ ) at around $8.1 \times 10^{4}$ orbital periods in the coorbital mode with argument $\phi=\lambda^{\star}-\lambda_{\mathrm{J}}+2 \Omega$ and center $\phi=180^{\circ}$ 
Fig. 2 Capture in the planar problem. The particle's semi-major axis $a$, eccentricity $e$ and resonant argument $\phi^{\star}=\lambda+\lambda_{\mathrm{J}}-2 \varpi$ are shown as a function of time in units of the planetary period. In the upper panel, the red solid curve is the planet's semi-major axis and the dotted blue line is the planet's Hill radius

Fig. 3 Capture in the three-dimensional problem with initial inclination $179.999^{\circ}$. The particle's semi-major axis $a$, eccentricity $e$ and inclination are shown as a function of time in units of the planetary period. Also shown are the possible resonant angles: $\phi=\lambda^{\star}-\lambda_{\mathrm{J}}+2 \Omega$, $\phi^{\star}=\lambda^{\star}-\lambda_{\mathrm{J}}-2 \varpi^{\star}$. In the upper panel, the red solid curve is the planet's semi-major axis and the dotted blue line is the planet's Hill radius
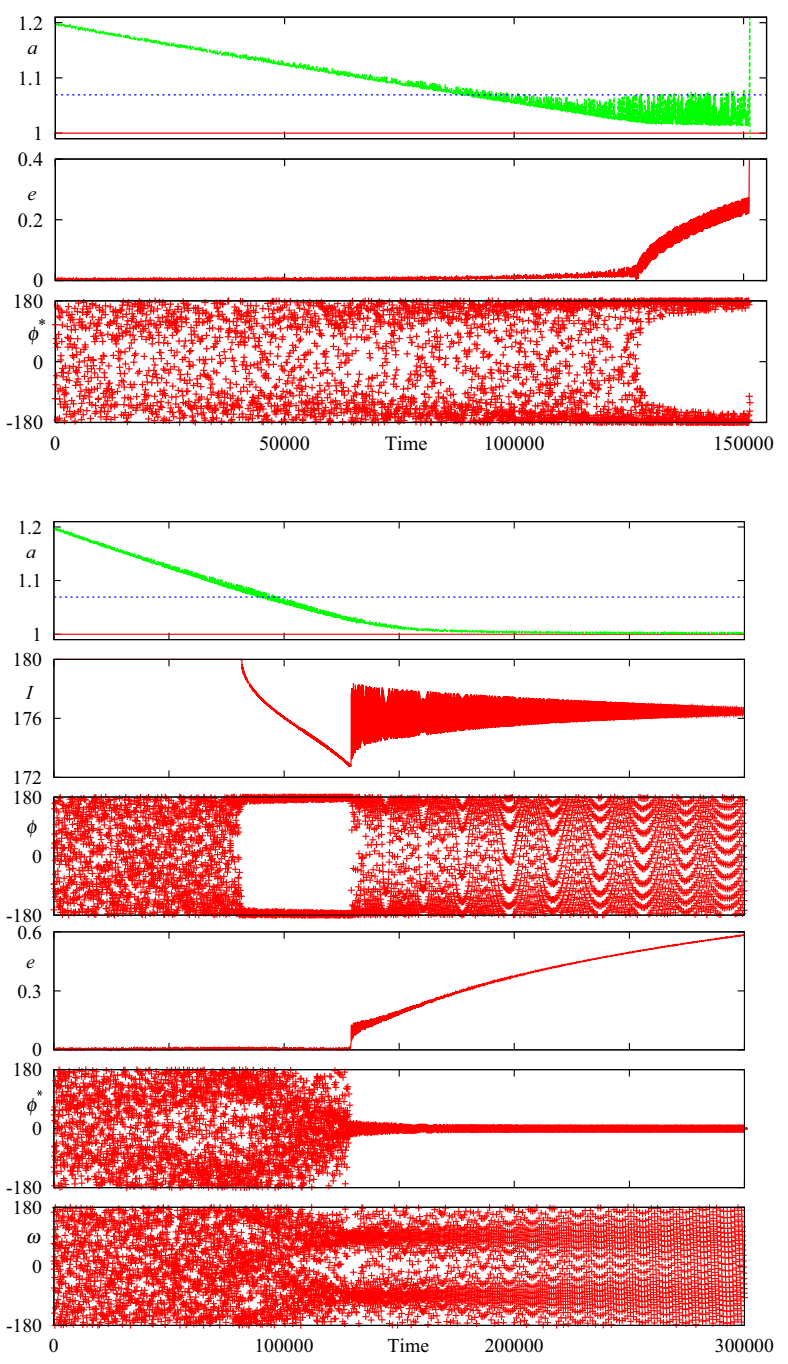

(mode R4) . As explained in Sect. 2, when written in terms of the standard orbital elements, the argument $\phi=\lambda-\lambda_{\mathrm{J}}$ is that of the standard prograde coorbital resonance. However, for retrograde motion the resonance behaves exactly like an inclination resonance witness the steady decrease (increase) of (relative) inclination whereas the eccentricity remains constant. Capture in coorbital mode R4 lasts about $5 \times 10^{4}$ orbital periods until the Kozai-Lidov resonance is triggered. In Fig. 4, we show how the Kozai-Lidov resonance affects 3D capture in the time interval between $1.25 \times 10^{5}$ and $1.3 \times 10^{5}$. Since the initial eccentricity is zero, $\omega$ circulates near the Kozai-Lidov centers at $\pm 90^{\circ}$ and $e \approx 0.01$. As the semi-major axis slowly decreases the topology of the secular phase-space changes and the particle gets trapped at the $\omega=-90^{\circ}$ and later at the $\omega=90^{\circ}$ centers (evidenced by the higher density of points in those regions). The libration island at $\omega=90^{\circ}$ gets displaced to higher eccentricity and eventually the orbit reaches the vicinity of the Kozai-Lidov separatrix associated with the $\omega=0$ and $\omega=180^{\circ}$ islands. The angle $\omega$ circulates again but now the eccentricity oscillates between 
Fig. 4 Kozai-Lidov resonance stage (between $1.25 \times 10^{5}$ and $1.3 \times 10^{5}$ ) from Fig. 3 (see text for details)

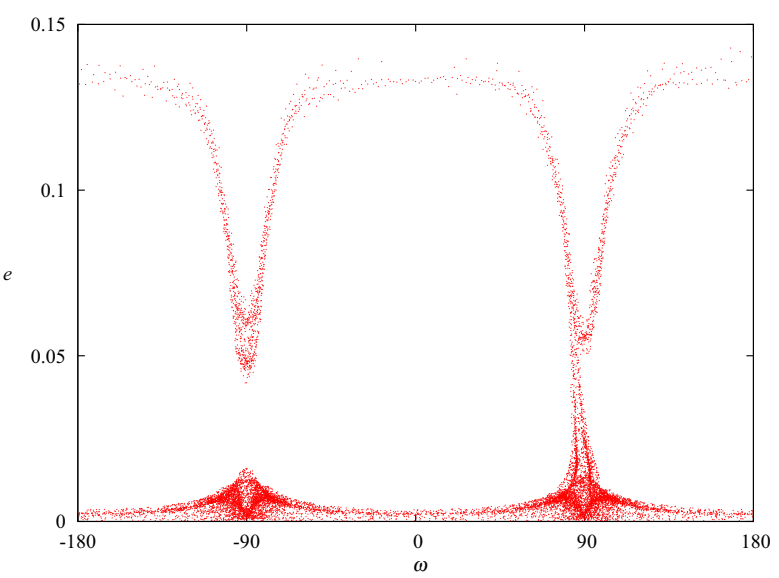

0.05 and 0.13 allowing capture in the coorbital mode with argument $\phi^{\star}=\lambda^{\star}-\lambda_{\mathrm{J}}-2 \varpi^{\star}$ and center $\phi^{\star}=0$ (mode R1). The particle remains trapped in coorbital mode R1 while the eccentricity increases steadily, the typical behavior of an eccentricity resonance. Collisions are unlikely until the particle's orbit reaches unit eccentricity. ${ }^{5}$

The presence of the Kozai-Lidov mechanism during mean motion resonance capture is a process that was observed in resonance capture at arbitrary inclination in our intensive $(\sim 600000)$ simulations of Paper II. In order to illustrate further its workings, we present another $3 \mathrm{D}$ simulation with identical initial conditions except for the initial inclination set to $175^{\circ}$ and a radial drift 10 times slower than previously. We increased relative inclination so as to give a stronger effect to inclination resonances. Similarly we slowed down the semimajor axis drift so as to allow the particle to explore a larger portion of the secular phase space. Figure 5 shows the evolution of the orbital elements and resonant angles from the capture in mode $\mathrm{R} 4\left(\phi=180^{\circ}\right)$ to the capture in mode $\mathrm{R} 1\left(\phi^{\star}=0\right)$. New features appear such as the temporary exit from mode R4 corresponding to brief KozaiLidov oscillation around $\pm 90^{\circ}$ as well as the long capture phase in the $\omega=0$ Kozai-Lidov resonance preceding capture in mode $\mathrm{R} 1$. Capture in the inclination-type retrograde resonance with centre $\phi=180^{\circ}$ (mode R4) has a relatively shorter duration (with respect to drift time) when initial inclination is decreased. We have checked that the three-stage capture mechanism is generic and independent of the semi-major axis drift rate as long as this is much slower than the libration period to allow for an adiabatic resonance crossing. Outwardly migrating particles with initially circular orbits interior to the planet do not get captured in the coorbital zone whether the retrograde configuration is plane or slightly inclined.

\section{Stability analysis for $2 \mathrm{D}$ configurations}

Stability analysis is an important tool to understand how capture occurs in the three-body problem. By charting parameter space, one is able to identify the possible dynamical routes that the particle's orbit may take to enter resonance. To this end, we numerically integrate the

5 If all bodies are treated as point-like objects and evolution is allowed to continue, no collision occurs. Instead orbital reversal takes place (Yu and Tremaine 2001). 
Fig. 5 Capture in the three-dimensional problem as in Fig. 3 but with initial inclination $175^{\circ}$ and radial drift 10 times slower than in previous simulations

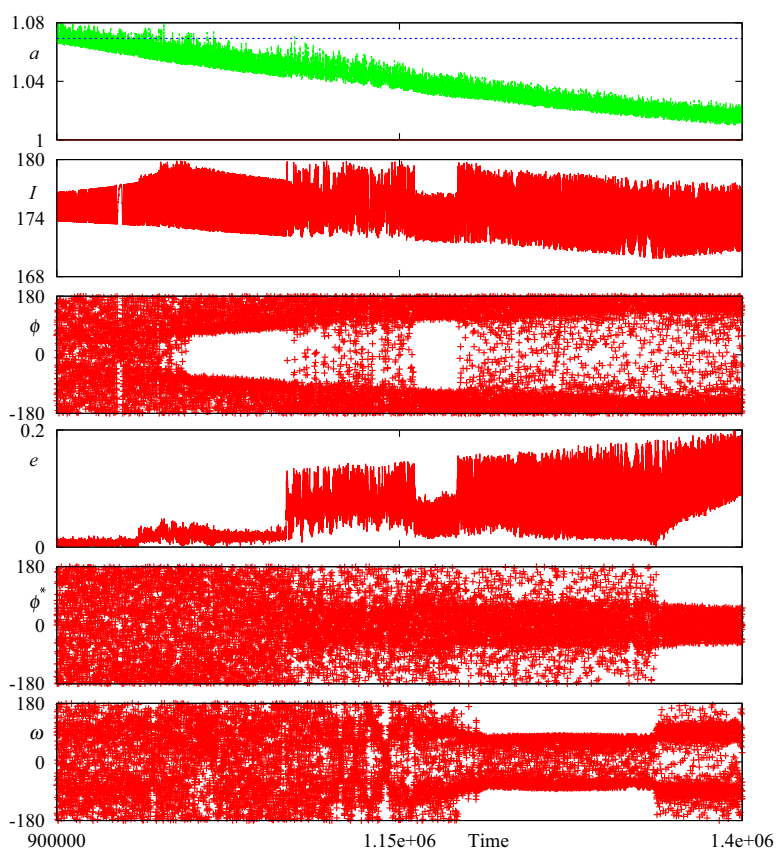

equations of motion of the circular restricted three-body problem consisting of a Sun-mass star, a Jupiter-like planet with a mass ratio $\mu=0.001$ and a massless particle, together with the variational equations and MEGNO equations (Cincotta and Simó 2000; Goździewski 2003 ) for $5 \times 10^{4}$ periods using a Burlisch-Stoer method with double precision arithmetics and tolerance $10^{-14}$. The mean MEGNO $\langle Y\rangle$ converges to 2 for regular orbits and increases at a rate inversely proportional to Lyapunov's time for chaotic orbits (Cincotta and Simó 2000; Goździewski 2003). We set the maximum mean MEGNO $\langle Y\rangle$ value for chaotic orbits at 8 in order to present stability maps with a high contrast between the regular and chaotic regions. The integration of each test particle's orbit is stopped before $5 \times 10^{4}$ periods if collision or escape occur: a collision occurs when the minimum distances to the primary or planet are less than 0.005 or 0.0005 , respectively (the unit distance is the Sun-Jupiter semi-major axis as in the previous section). These values correspond approximately to the solar and Jovian radii. Escape occurs when the barycentric distance exceeds the value 3 .

\subsection{Retrograde orbits}

The test particle's initial conditions for the coorbital retrograde resonance are: inclination $I=180^{\circ}$, semi-major axis in $\left(1-2 R_{\mathrm{H}}, 1+2 R_{\mathrm{H}}\right)$ varying at steps $0.05 R_{\mathrm{H}}$, eccentricity in $(0,1)$ varying at steps 0.01 , angles $\Omega=\omega=0$ where $\omega=\varpi-\Omega$, and $\phi^{\star}=\lambda-\lambda_{\mathrm{J}}-2 \omega=$ $0,180^{\circ}$. In Fig. 6 we show the MEGNO maps for configurations with $\phi^{\star}=0$ (top panel) and $\phi^{\star}=180^{\circ}$ (low panel).

Coorbital mode R1 where $\phi^{\star}$ librates around 0 occurs for eccentricity $e \gtrsim 0.1$. Close encounters with the planet occur at pericentre and apocentre for small eccentricity orbits (Fig. 1: top right) hence the pericentric and apocentric collision lines limit the resonance borders in Fig. 6 (top panel). The apocentric collision line (right hand side) is surrounded by 
Fig. 6 Stability map for the planar retrograde coorbital resonance with $\phi^{\star}=0$ (top panel) or $\phi^{\star}=180^{\circ}$ (low panel) when $\mu=0.001$. The symbol + marks initial conditions such that $\phi^{\star}$ librates with amplitude less than $50^{\circ}($ black +$)$ or larger than $50^{\circ}($ gray +$)$. The black lines represent collision at pericentre (left hand side) or apocentre (right hand side). The red circles indicate initial conditions of orbits shown in Fig. 1
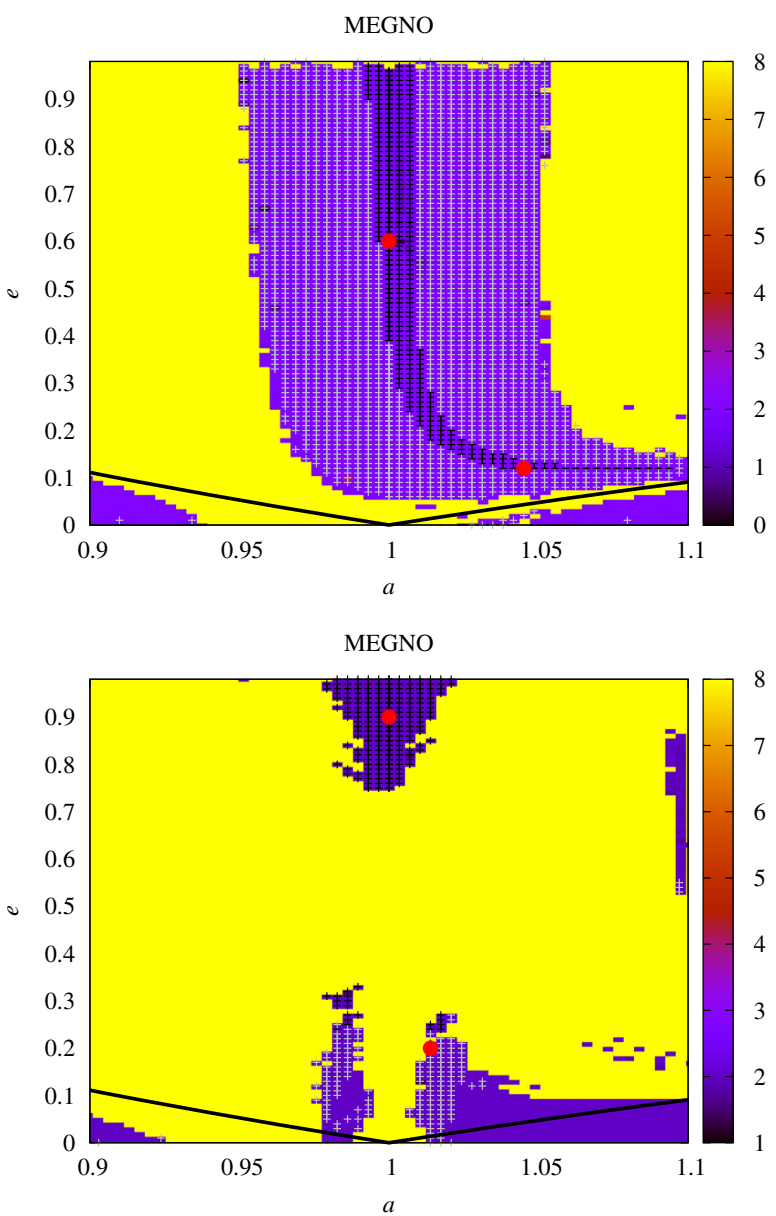

a thin chaotic layer which extends to $a>1.1$, separating the outer circulating region from the librating region (mode R1).

The coorbital modes where $\phi^{\star}$ librates around $180^{\circ}$ occur at eccentricity $e \gtrsim 0.7$ and $1.02 \gtrsim a \gtrsim 0.98$ (mode $\mathrm{R} 2$ ), or eccentricity $e \lesssim 0.3$ in the semi-major axis ranges $0.99 \gtrsim$ $a \gtrsim 0.98$ and $1.03 \gtrsim a \gtrsim 1.01$ (mode $\mathrm{R} 3$ ). The orbits in the vicinity of the resonance centers in Fig. 6 (low panel) are close to the collision separatrix located midway between pericentre and apocentre (Fig. 1: low right) but collisions with the planet are avoided. Repeated close encounters with the planet significantly disturb the osculating orbital elements which implies that these are not adequate to identify the resonance center at $\phi^{\star}=180^{\circ}$, the method of surfaces of section being more appropriate for this task (Paper I).

The resonance regions in Fig. 6 are surrounded by chaotic layers except for low eccentricity outer orbits with $\phi^{\star}$ librating around $180^{\circ}$ (mode R3 in the low panel). This feature explains why circular planar retrograde orbits migrating from the outer region towards the planet are always captured in coorbital mode R3 while those migrating from the inner region are never captured as seen in Sect. 3. 


\subsection{Prograde orbits}

The test particle's initial conditions for the coorbital prograde resonance are identical to the retrograde case except for the inclination $I=0$, and $\phi=\lambda-\lambda_{\mathrm{J}}=0,60^{\circ}, 180^{\circ}$. In Fig. 7 we show the MEGNO maps for configurations with $\phi=0$ (top panel), $\phi=60^{\circ}$ (mid panel) and $\phi=180^{\circ}$ (low panel).

Figure 7 (top panel) shows region of libration of RS (retrograde-satellite) orbits ( $\phi$ librates around 0 ) at $e \gtrsim 0.2$. Figure 7 (mid panel) shows regions of libration of T (tadpole) orbits $(\phi$ librates around $\pm 60^{\circ}$ ) at $e \lesssim 0.4$ and RS orbits at $e \gtrsim 0.6$. Figure 7 (low panel) shows that $\mathrm{H}$ (horseshoe) orbits $\left(\phi\right.$ librates around $\left.180^{\circ}\right)$ are only possible at $e \gtrsim 0.8$ when the mass ratio is $\mu=0.001$.

All resonance regions in Fig. 7 are surrounded by thick chaotic layers which extend over the entire range of eccentricities. Therefore, planar prograde orbits migrating towards the planet cannot be smoothly captured in the coorbital resonance as seen in Paper II. Instead, temporary capture due to chaotic scattering may occur.

\section{Stability analysis for 3D configurations}

The three-dimensional coorbital resonance is much richer than its planar counterpart (Namouni 1999; Namouni et al. 1999). In addition to the prograde corbital modes, tadpole $(\mathrm{T})$, retrograde satellite $(\mathrm{RS})$ and horseshoe $(\mathrm{H})$, retrograde satellites combine with tadpoles or horseshoes to form the compound orbits, H-RS, RS-H, T-RS, RS-T and T-RS-T. The third dimension also influences significantly orbital nature in another way. Whereas proper eccentricity is a secular constant in the planar configuration, it is subject to (possibly large) secular time variations along with the inclination giving rise to orbital transitions between the various libration modes as well as the appearance of the Kozai-Lidov resonance.

Owing to this particularly complex nature of the three-dimensional coorbital problem, in order to understand the basic features of resonance capture we will not attempt to identify coorbital mode transitions or compound orbits. We start our exploration by setting the particle's semi-major axis to unity. Eccentricity and inclination are chosen in a grid with $e$ between 0 and 0.99 with a step of 0.01 and $I$ between $5^{\circ}$ and $180^{\circ}$ with a step of $5^{\circ}$. The initial angles were ${ }^{6} \phi=0,60^{\circ}, 180^{\circ}$ and $\omega=0,90^{\circ}$. In Table 1 we show the initial values of $\phi^{\star}=\phi-2 \omega$ for each $\phi$ and $\omega$. The choice of initial angles $\phi$ and $\phi^{\star}$ is set to cover the coorbital configurations of the planar problem that we explored earlier. From Fig. 7 prograde coorbitals are those in the RS mode $(\phi \sim 0)$; T mode $\left(\phi \sim \pm 60^{\circ}\right)$; $\mathrm{H}$ mode $\left(\phi \sim 180^{\circ}\right)$. From Fig. 6 retrograde coorbitals at $a=1$ are: mode R1 $\left(\phi^{\star} \sim 0\right)$; mode R2 $\left(\phi^{\star} \sim 180^{\circ}\right)$. We set initially $\omega=0,90^{\circ}$ as these are the possible Kozai-Lidov libration centers.

For each initial condition in our grid, we computed a chaos indicator (mean MEGNO $\langle Y\rangle)$ and the libration amplitudes of the angles $\phi, \phi^{*}=\phi-2 \omega$ and $\omega$ that serve to identify resonant orbits.

In Figs. 8 and 9 we see that stable regions are mostly associated with resonant orbits such that the angles $\phi$ or $\phi^{*}$ librate, signaled respectively by triangles or circles (black symbols indicate libration amplitude less than $50^{\circ}$ while gray symbols indicate libration amplitude larger than $50^{\circ}$ ). The observed resonant modes are:

- RS: libration of $\phi$ around 0 at moderate to large eccentricity and $I \lesssim 100^{\circ}$;

6 The 1:1 resonance Hamiltonian is invariant with respect to the transformation $(\phi,-\omega) \rightarrow(-\phi, \omega)$. The CR3BP is invariant with respect to the transformation $\omega \rightarrow \omega+180^{\circ}$ and with respect to changes in $\Omega$. 
Fig. 7 Stability map for the planar prograde coorbital resonance with $\phi=0$ (top panel), $\phi=60^{\circ}$ (mid panel) or $\phi=180^{\circ}$ (low panel) when $\mu=0.001$. The symbol + marks initial conditions such that $\phi$ librates with amplitude less than $50^{\circ}($ black +$)$ or larger than $50^{\circ}($ gray +$)$. The black lines represent collision at pericentre (left hand side) or apocentre (right hand side)
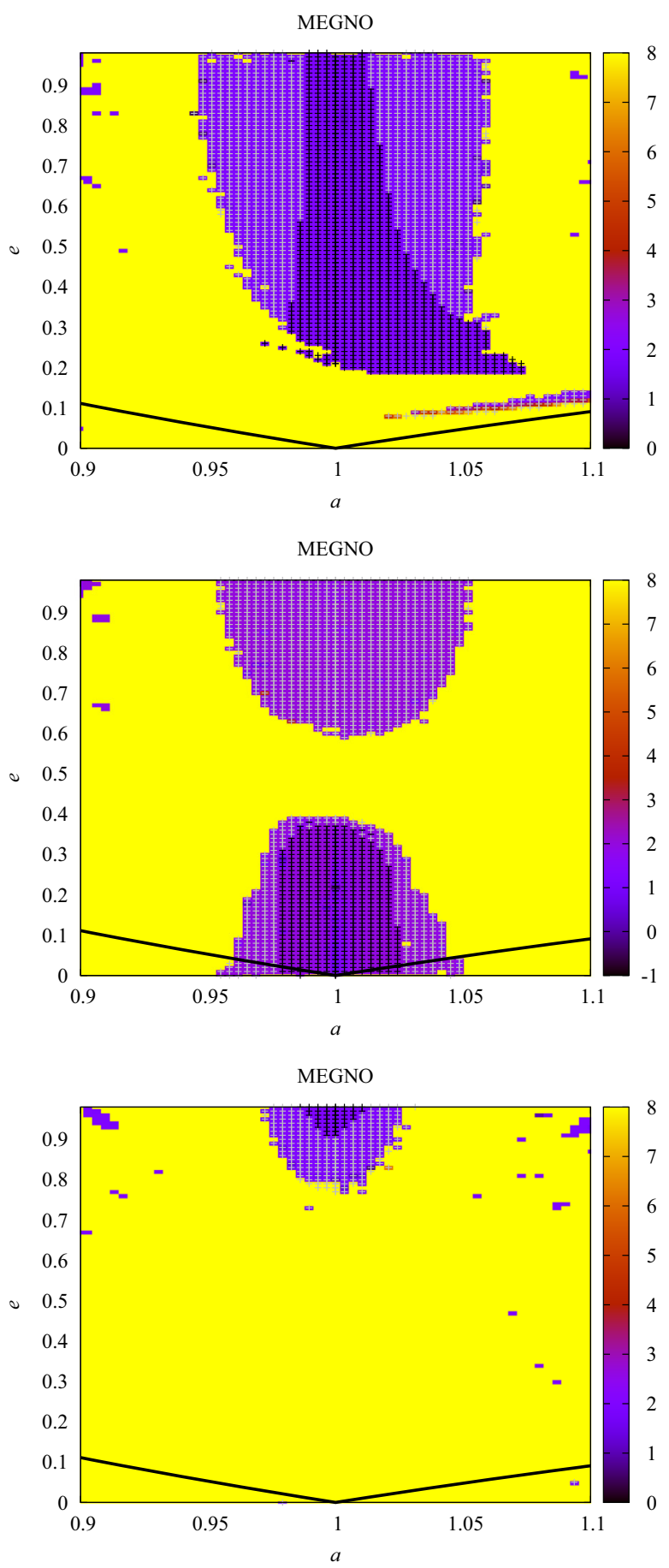
Table 1 Initial angles $\phi, \phi^{\star}, \omega$ for simulations shown in Figs. 6 and 7

\begin{tabular}{lll}
\hline & $\omega=0$ & $\omega=90^{\circ}$ \\
\hline$\phi=0$ & $\phi^{\star}=0$ & $\phi^{\star}=180^{\circ}$ \\
$\phi=60^{\circ}$ & $\phi^{\star}=60^{\circ}$ & $\phi^{\star}=240^{\circ}$ \\
$\phi=180^{\circ}$ & $\phi^{\star}=180^{\circ}$ & $\phi^{\star}=0$ \\
\hline
\end{tabular}

- T: asymmetric libration of $\phi$ around $\pm 60^{\circ}$ at $e \lesssim 0.4$ and $I \lesssim 60^{\circ}$;

- R4: libration of $\phi$ around $180^{\circ}$ at $e<0.3$ and $120^{\circ} \lesssim I<180^{\circ}$;

- H: libration of $\phi$ around $180^{\circ}$ at $e>0.8$ and $I \lesssim 70^{\circ}$;

- R1: libration of $\phi^{*}$ around 0 at wide range of eccentricities and $I>90^{\circ}$;

- R2: libration of $\phi^{*}$ around $180^{\circ}$ at $e>0.7$ and $I \gtrsim 120^{\circ}$.

Due to output sampling some circulating orbits are wrongly identified as high amplitude librating orbits. This explains the isolated gray circles inside the RS regions (Figs. 6, 7: top panels) and inside the T region (Fig. 6: mid panel).

The Kozai-Lidov resonance (libration of $\omega$ ) is identified in Figs. 8 and 9 with dark + symbols (libration amplitude less than $50^{\circ}$ ) and light + symbols (libration amplitude larger than $50^{\circ}$ ) and may also ensure stability without requiring the libration of $\phi$ and $\phi^{*}$. For prograde configurations, these are the passing $(\mathrm{P})$ orbits that periodically transit to the compound H-RS and RS-H modes on secular time scales (Namouni 1999; Namouni et al. 1999).

Modes RS, T and $\mathrm{H}$ are present in the 2D prograde coorbital problem. Modes R1 and R2 are present in the 2D retrograde coorbital problem as shown in Paper I. Mode R4 only exists in the 3D coorbital problem and is the retrograde analogue to an horseshoe orbit (mode $\mathrm{H}$ ) since $\phi$ librates around $180^{\circ}$. There are regions where both $\phi$ and $\omega$ (hence $\phi^{*}$ ) librate which are associated with equilibria of the 3D coorbital model. The latter orbits are identified in Figs. 6 and 7 by an overlap of the triangles, circles and + symbols.

When $\phi=0$ and $\omega=0$ initially (Fig. 8: top panel) the only possible resonant orbits are: mode RS and mode R1 at moderate to large eccentricities. The transition between RS and R1 modes does not occur exactly at $I=90^{\circ}$ (e.g. at $e=0.6, \mathrm{RS}$ orbits have $I \leq 95^{\circ}$ while R1 orbits have $I>95^{\circ}$ ).

When $\phi=60^{\circ}$ and $\omega=0$ initially (Fig. 8: mid panel) modes RS and R1 are present at moderate to large eccentricity, and mode T (tadpole orbits) occurs at $e \lesssim 0.4$ and $I \lesssim 60^{\circ}$. Mode R4 orbits where $\phi$ librates around $180^{\circ}$ occur at retrograde inclination $130^{\circ} \lesssim I<$ $180^{\circ}$ and $e<0.3$ (indeed a careful look at this panel reveals a tiny mode $\mathrm{R} 4$ region for nearly circular retrograde orbits slightly off the plane). The Kozai-Lidov resonance around $\omega=0$ occurs at the border of the region of libration in mode R4.

When $\phi=180^{\circ}$ and $\omega=0$ initially (Fig. 8: low panel), mode R4 occurs at $e<0.3$ and $I \sim 120^{\circ}$. Horseshoe orbits (where $\phi$ librates around $180^{\circ}$ ) with small eccentricities and low inclinations are unstable at mass ratio 0.001 due to the effect of close encounters with the planet (Fig. 7: low panel). However, such close encounters are less disruptive when the motion is retrograde (as these occur at higher relative velocities) which explains the stability of mode R4 orbits. At large eccentricities there are stable prograde horseshoe orbits (mode $\mathrm{H}$ ) which avoid close encounters with the planet by being always near opposition with respect to the star. Large eccentricity retrograde orbits in mode R2 are also stable. The Kozai-Lidov resonance occurs outside the region of libration of $\phi$ or $\phi^{\star}$ and thus concerns passing $(\mathrm{P})$ orbits. In particular, retrograde and nearly-polar orbits in that resonance are stable. 
Fig. 8 Stability maps for coorbital resonance in CR3BP: initial conditions $a=1, \omega=0$ and $\phi=0$ (top panel), $\phi=60^{\circ}$ (mid panel), $\phi=180^{\circ}$ (low panel). The symbol + marks initial conditions such that $\omega$ librates with amplitude less than $50^{\circ}($ dark +$)$ or larger than $50^{\circ}$ $($ light +$)$. The triangles (circles) mark initial conditions such that $\phi\left(\phi^{\star}\right)$ librate with amplitude less than $50^{\circ}$ (black) or larger than $50^{\circ}$ (gray)

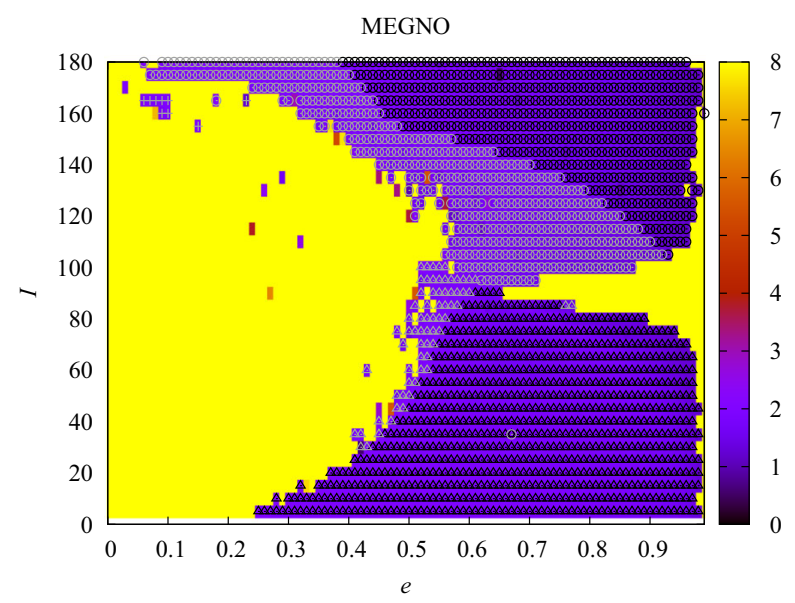

MEGNO
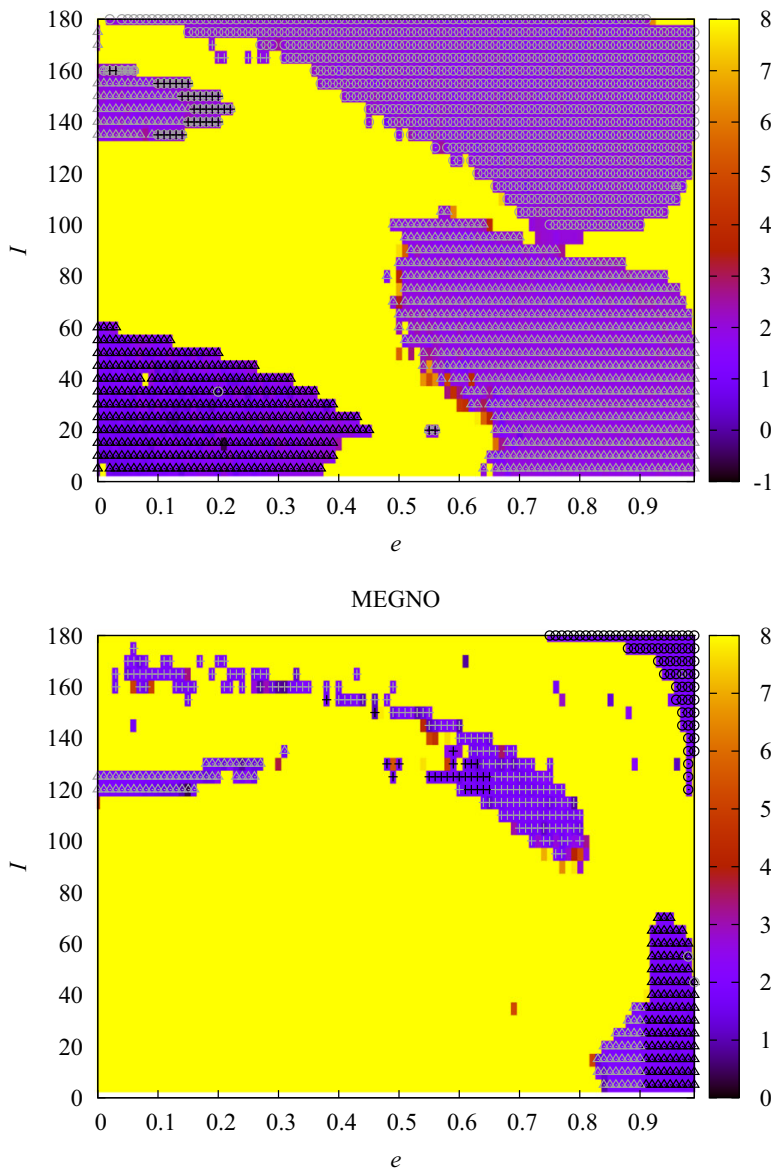
Fig. 9 Stability maps for coorbital resonance in CR3BP: initial conditions $a=1, \omega=90^{\circ}$ and $\phi=0$ (top panel), $\phi=60^{\circ}$ (mid panel), $\phi=180^{\circ}$ (low panel). The symbol + marks initial conditions such that $\omega$ librates with amplitude less than $50^{\circ}($ dark +$)$ or larger than $50^{\circ}$ (light +$)$. The triangles (circles) mark initial conditions such that $\phi\left(\phi^{\star}\right)$ librate with amplitude less than $50^{\circ}$ (black) or larger than $50^{\circ}$ (gray)
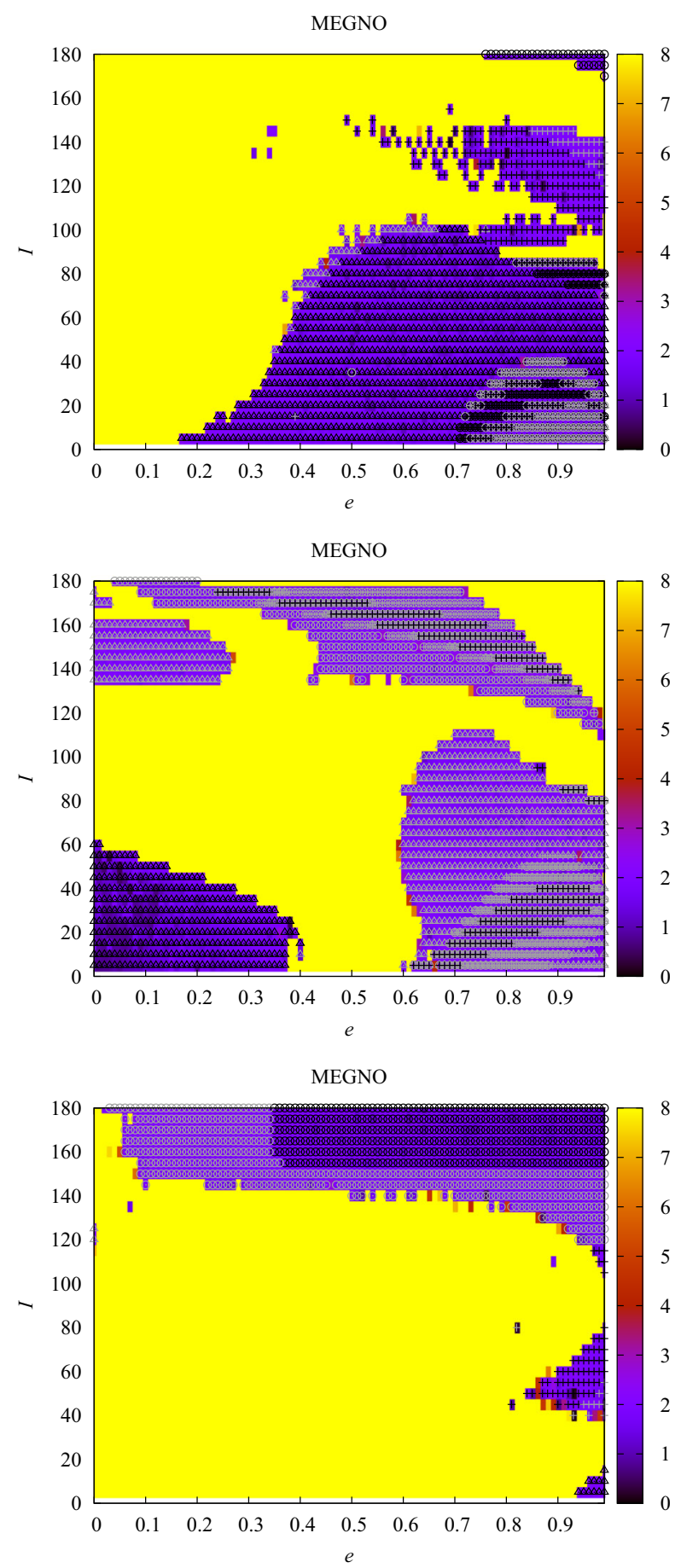
Fig. 10 Top panel: stability map in $(a, i)$ grid for initial conditions $\omega=0, \phi=180^{\circ}, e=0$. The triangles mark initial conditions such that $\phi$ librates around $180^{\circ}$ with amplitude less than $50^{\circ}$ (black) or larger than $50^{\circ}$ (gray). Low panel: mode R4 orbit close to exact resonance viewed in 3D synodic frame. Initial conditions $I=175^{\circ}$, $\phi=180^{\circ}, \omega=0, a=1.04507$ and $e=0$. The star (Sun) is located at $(0,0,0)$ and the planet (Jupiter) at $(1,0,0)$

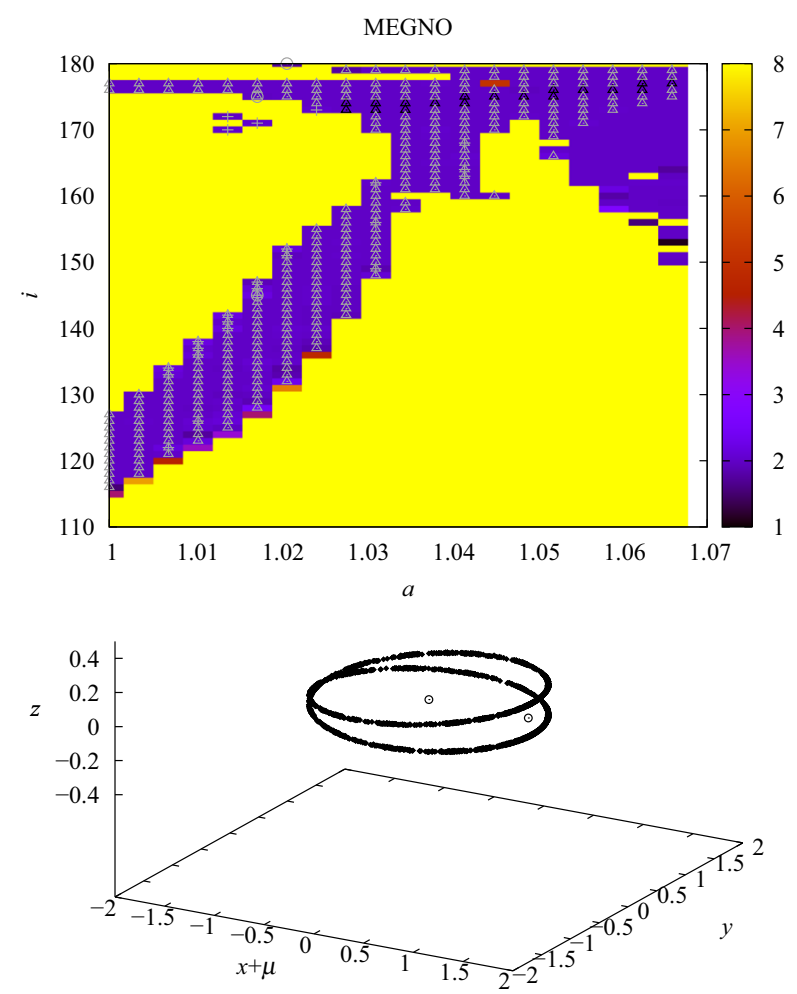

When $\phi=0$ and $\omega=90^{\circ}$ initially (Fig. 9: top panel) mode RS is present at $e \gtrsim 0.2$ and $I \lesssim 100^{\circ}$, while mode R2 occurs at $e>0.7$ and $I \gtrsim 170^{\circ}$. The Kozai-Lidov resonance is present at $I \lesssim 30^{\circ}$ inside the region of libration in mode RS, and also at large eccentricities and inclination $100^{\circ} \lesssim I \lesssim 140^{\circ}$. Equilibria of the 3D coorbital problem occur when there is simultaneous libration in mode RS along with the Kozai-Lidov resonance since all angles $\left(\phi, \phi^{\star}\right.$ and $\left.\omega\right)$ are stationary.

When $\phi=60^{\circ}$ and $\omega=90^{\circ}$ initially (Fig. 9: mid panel) resonant orbits include mode RS at $e>0.6$ and $I \lesssim 110^{\circ}$, mode T (tadpole orbits) at $e \lesssim 0.4$ and inclination $I \lesssim 60^{\circ}$, mode $\mathrm{R} 4$ at $e<0.3$ and inclination $130^{\circ}<I<180^{\circ}$, mode R1 at a wide range of eccentricities and $I \gtrsim 110^{\circ}$. The Kozai-Lidov resonance is present inside the regions of libration in modes $\mathrm{RS}$ and $\mathrm{R} 1$.

When $\phi=180^{\circ}$ and $\omega=90^{\circ}$ initially (Fig. 9: low panel) possible resonant orbits are mode $\mathrm{H}$ at $e \gtrsim 0.95$ and $I<20^{\circ}$, and mode $\mathrm{R} 1$ at a wide range of eccentricities and $I>100^{\circ}$. The Kozai-Lidov resonance occurs at $e>0.8$ and $40^{\circ} \lesssim I \lesssim 80^{\circ}$.

According to Fig. 6 mode R3 is possible only at $a>1$ or $a<1$. In order to explore the retrograde region at small eccentricities and to assess the stability of mode R3 off the plane we computed stability maps with initial conditions: $\phi=180^{\circ}, \omega=0$, semi-major axis $1 \leq a \leq 1+R_{H}$ varying at steps $0.05 R_{H}$, inclination $90^{\circ} \leq I \leq 180^{\circ}$ varying at $1^{\circ}$ steps, and fixed eccentricity: $0 \leq e \leq 0.2$. These initial conditions cover the regions of libration associated with mode R3 (Fig. 6: low panel) and mode R4 (Fig. 8: mid an low panels). In Fig. 10 (top panel) we show the case with initial $e=0$. We see that mode R3 ( $\phi^{\star}$ librating around $180^{\circ}$ at small eccentricities), identified by a single gray circle located at $a \approx 1.02$ 
and $I=180^{\circ}$, is present only in the retrograde planar problem. At inclinations slightly off the plane this is replaced by mode R4 (retrograde orbit with $\phi$ librating around $180^{\circ}$ ) whose resonance centre is identified by black triangles in Fig. 10 (top panel). In Fig. 10 (low panel) we show synodic frame $3 \mathrm{D}$ view of a mode $\mathrm{R} 4$ orbit corresponding to initial conditions $I=175^{\circ}, \phi=180^{\circ}, \omega=0, a=1.04507$ and $e=0$. This is close to exact retrograde resonance with $\phi=180^{\circ}$ and corresponds to the orbit in Fig. 2 approximately midway within the capture episode in mode R4. At the planet's vicinity the orbit is either above or below the $(x, y)$ plane, thus avoiding collision. The shape in the synodic frame is closer to mode $\mathrm{R} 1\left(\phi^{\star}=0\right.$, as in Fig. 6: top right panel) than mode R3 $\left(\phi^{\star}=180^{\circ}\right.$, as in Fig. 6: low right panel)

\section{Conclusion}

We investigated the mechanism of coorbital capture for retrograde orbits migrating towards a Jupiter mass planet. This study was motivated by our previous results concerning resonance capture (Paper II) which showed that retrograde orbits were more likely to get captured in resonance than prograde orbits. In addition, we reported that retrograde orbits migrating inwards with small relative inclination were more likely to be captured in the coorbital resonance. In particular, circular orbits with $I=180^{\circ}$ were captured into coorbital mode R3 with a $100 \%$ efficiency (Paper II). Here, we presented 2D stability maps of the retrograde coorbital resonance which show that smooth (non chaotic) migration capture is only possible into mode R3 for nearly circular outer orbits.

We explained the differences between the capture mechanism and end states for retrograde planar orbits $\left(I=180^{\circ}\right.$ exactly) compared with retrograde orbits inclined with respect to the planet's orbital plane. While planar retrograde orbits are captured in mode R3 $\left(\phi^{\star}=180^{\circ}\right)$, for retrograde orbits only slightly off the plane capture occurs in a three stage process: first mode R4 $\left(\phi=180^{\circ}\right)$, an inclination-type resonance that does not occur in the 2D case, then the Kozai-Lidov resonance, and finally mode R $1\left(\phi^{\star}=0^{\circ}\right)$, an eccentricity-type resonance. Our large scale $(\sim 600000)$ simulations from Paper II showed that 3D capture into other resonances also follows a three-stage process.

We computed stability maps in order to identify the possible stable coorbital modes for arbitrary inclination. Such maps indicate that retrograde modes R1 and R2 persist in the three-dimensional problem while mode R3 seems to only be possible in two dimensions, the case studied in Paper I. This explains why capture in mode R3 for initially circular orbits occurs only when $I=180^{\circ}$ exactly. An additional coorbital mode, R4, a retrograde analogue of horseshoe-type orbits, appears at low eccentricity and retrograde inclination $I<180^{\circ}$. As expected, the Kozai-Lidov resonance is present in the 3D coorbital problem. Moreover, the simulations of retrograde coorbital capture described here and in Paper II show that the Kozai-Lidov mechanism is key to the increases in eccentricity necessary for final capture into mode R1.

We saw that retrograde coorbital resonance capture in 3D is essentially different from the $2 \mathrm{D}$ case even for tiny relative inclinations. This may be a peculiarity of the retrograde coorbital resonance (we leave the detailed investigation of other resonances to a future work). Moreover, we showed that stable coorbital modes exist at all inclinations, including retrograde and polar obits. Real examples of these orbits have not yet been found. Our results imply that high inclination coorbital companions of the solar system planets are a viable dynamical possibility and encourage future searches for such objects. 
Acknowledgments We thank Nelson Callegari Jr. for assistance with computational resources.

\section{References}

Cincotta, P.M., Simó, C.: Simple tools to study global dynamics in non-axisymmetric galactic potentials-I. A\&AS 147, 205-228 (2000). doi:10.1051/aas:2000108

Goździewski, K.: Stability of the HD 12661 planetary system. Astron. Astrophys. 398, 1151-1161 (2003). doi:10.1051/0004-6361:20021713

Mikkola, S., Brasser, R., Wiegert, P., Innanen, K.: Asteroid 2002 VE68, a quasi-satellite of Venus. Mon. Not. R. Astron. Soc. 351, L63-L65 (2004). doi:10.1111/j.1365-2966.2004.07994.x

Morais, M.H.M., Giuppone, C.A.: Stability of prograde and retrograde planets in circular binary systems. Mon. Not. R. Astron. Soc. 424, 52-64 (2012). doi:10.1111/j.1365-2966.2012.21151.x. arXiv:1204.4718

Morais, M.H.M., Namouni, F.: Asteroids in retrograde resonance with Jupiter and Saturn. Mon. Not. R. Astron. Soc. 436, L30-L34 (2013a). doi:10.1093/mnrasl/slt106. arXiv:1308.0216

Morais, M.H.M., Namouni, F.: Retrograde resonance in the planar three-body problem. Celest. Mech. Dyn. Astron. 117, 405-421 (2013b). doi:10.1007/s10569-013-9519-2. arXiv:1305.0016

Murray, C.D., Dermott, S.F.: Solar System Dynamics. Cambridge University Press, Cambridge (1999)

Namouni, F.: Secular interactions of coorbiting objects. Icarus 137, 293-314 (1999). doi:10.1006/icar.1998. 6032

Namouni, F., Morais, M.H.M.: Resonance capture at arbitrary inclination. Mon. Not. R. Astron. Soc. 446, 1998-2009 (2015). doi:10.1093/mnras/stu2199. arXiv:1410.5383

Namouni, F., Christou, A.A., Murray, C.D.: Coorbital dynamics at large eccentricity and inclination. Phys. Rev. Lett. 83, 2506-2509 (1999). doi:10.1103/PhysRevLett.83.2506

Nesvorný, D., Thomas, F., Ferraz-Mello, S., Morbidelli, A.: A perturbative treatment of the co-orbital motion. Celest. Mech. Dyn. Astron. 82, 323-361 (2002). doi:10.1023/A:1015219113959

Yu, Q., Tremaine, S.: Resonant Capture by Inward-migrating Planets. Astron. J. 121, 1736-1740 (2001). doi:10.1086/319401. arXiv:astro-ph/0009255 